\title{
Experimental accuracy assessment of different measuring sensors on workpieces with varying properties
}

\author{
Rauf Oezden ${ }^{1, *}$, Metin Aclan ${ }^{1}$ and Saliba Danho ${ }^{2}$ \\ ${ }^{1}$ Technical University of Cluj-Napoca, Ph.D. Stud., Eng., Department for Manufacturing \\ Technology, Romania \\ 2 Johannes Gutenberg University Mainz, Ph.D. Stud., Eng., Germany
}

\begin{abstract}
The paper presents an investigation of measuring sensors in the manufacturing metrology. Industrial workpieces with varying surface-, form- and material properties will be investigated. The paper provides also an overview about the accuracy and reproducibility of measuring sensors by usual plastic- and metal parts. The parts will be much more complex in their geometry and the quality requirement are stronger monitored. Thus, the expectations in the manufacturing metrology are set higher.
\end{abstract}

\section{Introduction}

Nowadays, technical products consisting of several individual parts are very complex. Today's machines and tools are also capable of producing within tighter tolerance limits. Often it is about saving weight and the material. As a result, the parts produced become more filigree and the quality requirements higher [1].

In the measurement laboratory, the initial dimensional measurement of all individual single parts from all product areas, is then carried out. The geometric properties are measured with high-precision measuring equipment and checked according to drawing specifications and the conformity. In the maturing phase, the development process is supported by services up to the release of the individual products.

When carrying out a measurement task, the setting parameters of a measuring device make a considerable contribution to the measurement error. The operator decides whether to perform the measurements tactile or optical. For example, each operator selects different types of lighting for the same measuring task. These influences of the operator, has the disadvantage that different measured values are not reproducibility. Influences caused by measuring sensors and different workpiece properties must be investigated to ensure smooth production processes.

The paper examined the technical equipment of the multisensor coordinate measuring machines with different types of lighting (transmitted light variant and reflected light variant) and the tactile sensor. Furthermore, was also considered the computed tomography. In addition, different test objects contribute to the accuracy and reproducibility of the process.

\footnotetext{
* Corresponding author: rauf.oezden@outlook.com
} 


\section{Experimental implementations to optimize the application in industrial metrology}

The following conditions were defined for the experimental investigations and for the highest possible degree of automation:

- Test objects and measurement sensors must be free of systematic measurement influences before each measurement [2].

- An alignment for the objects create influences, because the datum area will be detected differently. That's why the alignment for the test objects has been neglected. In each test object are two holes milled. The direct distance to the depending surface is measured. The middle point from each hole should be determined by the Gaussian method.

- The test objects are tested in a saturated state to avoid the water absorption especially of the plastic objects (leads to changing the geometry) and to ensure that there is hardly any influence on the measurement results. Furthermore, the objects are continuously stored in a climatic chamber and are set at reference conditions $20 \mathrm{C}^{\circ}$ for the metal objects [2], $23 \mathrm{C}^{\circ}$ and $50 \%$ rel. humidity for the plastic objects [3].

- In order to eliminate operator influences, programs are written for the measurements, so that the same procedure is always followed over a long period of time and the measured values are obtained under the same conditions with the same strategy.

- Because many different magnifications are integrated for the evaluations by different types of lighting, it's important for traceability, that the maximum possible magnification is set, in which the hole can still be measured in the image (whole hole in image visible).

\section{Reproducibility check by evaluation on different workpieces}

The diameters and distances between two holes, one on a metal part and two plastic parts (here for examination in the color black and orange) were checked. The examined objects (metal and plastic) has different distances. The objects represent the average sizes of the produced workpieces of metal and plastic material. Table 1 presents the first experiment on the metal part. All values listed in the tables below are mean values from several measurements each, so that random runaways are largely excluded.

Table 1. Evaluation on material steel.

\begin{tabular}{|c|c|c|c|}
\hline \multicolumn{4}{|c|}{ Metal part - Steel / 10.5 x Magnification } \\
\hline IIlumination / light intensity & $\begin{array}{c}\text { Siameter left } \\
{[\mathrm{mm}]}\end{array}$ & $\begin{array}{c}\text { Diameter right } \\
{[\mathrm{mm}]}\end{array}$ & Distance [mm] \\
\hline Transmitted light = 15\% & 2,016 & 2,001 & 64,362 \\
\hline Transmitted light = 20\% & 2,016 & 2,001 & 64,363 \\
\hline Transmitted light = 27\% & 2,017 & 2,002 & 64,362 \\
\hline Bright field reflected light =5\% & 2,015 & 2,005 & 64,364 \\
\hline Tactile sensor & 2,015 & 2,001 & 64,362 \\
\hline Computed tomography & 2,044 & 2,022 & 64,407 \\
\hline MAX-MIN & 0,029 & 0,021 & 0,045 \\
\hline
\end{tabular}

The biggest systematic measurement errors are reached by the computed tomography (red marked in Table 1). The examination was carried out with an X-ray voltage of $130 \mathrm{kV}$ and thus explains the significant deviations from Table 1 on the computer tomography. The material of the metal part has a thickness of about $6 \mathrm{~mm}$ and therefore the X-ray tension would not be ideal designed. The conclusion for this, is that the computed tomography is not 
to be recommended, if the rays conditions cannot be followed and are important for the compliance of the reproducibility. The result is a classical systematic measurement error. The following Table 2 is presented the rays conditions for different used material and -thickness. This can be used as a guideline for industrial measurements by computed tomography.

Table 2. Links between tube voltage, workpiece size and workpiece material [4].

\begin{tabular}{|c|c|c|c|c|c|}
\hline X-ray voltage & $130 \mathrm{kV}$ & $150 \mathrm{kV}$ & $190 \mathrm{kV}$ & $225 \mathrm{kV}$ & $450 \mathrm{kV}$ \\
\hline Steel & $\$ 5 \mathrm{~mm}$ & $\$ 8 \mathrm{~mm}$ & $\$ 25 \mathrm{~mm}$ & $\$ 40 \mathrm{~mm}$ & $\$ 70 \mathrm{~mm}$ \\
\hline Aluminium & $\$ 30 \mathrm{~mm}$ & $\$ 50 \mathrm{~mm}$ & $\$ 90 \mathrm{~mm}$ & $\$ 150 \mathrm{~mm}$ & $\$ 250 \mathrm{~mm}$ \\
\hline Plastic & $\$ 90 \mathrm{~mm}$ & $\$ 130 \mathrm{~mm}$ & $\$ 200 \mathrm{~mm}$ & $\$ 250 \mathrm{~mm}$ & $\$ 450 \mathrm{~mm}$ \\
\hline
\end{tabular}

In Table 3, the next and subsequent experiment is demonstrated on the black plastic part.

Table 3. Evaluation on the black plastic material.

\begin{tabular}{|c|c|c|c|}
\hline \multicolumn{4}{|c|}{ Plastic part - Black / 8 x Magnification } \\
\hline Illumination / Light intensity & $\begin{array}{c}\text { Diameter left } \\
{[\mathrm{mm}]}\end{array}$ & $\begin{array}{c}\text { Siameter right } \\
{[\mathrm{mm}]}\end{array}$ & Distance [mm] \\
\hline \hline Transmitted light $=\mathbf{1 5 \%}$ & 3,493 & 3,488 & 70,596 \\
\hline \hline Transmitted light = 20\% & 3,492 & 3,488 & 70,595 \\
\hline Transmitted light = 27\% & 3,494 & 3,489 & 70,596 \\
\hline Bright field reflected light =5\% & 3,484 & 3,476 & 70,592 \\
\hline Tactile sensor * & 3,493 & 3,513 & 70,612 \\
\hline Computed tomography & 3,495 & 3,483 & 70,602 \\
\hline MAX-MIN & 0,011 & 0,037 & 0,020 \\
\hline
\end{tabular}

The large differences caused by the tactile measurement can be described in Figure 1. Since the diameter is measured through the middle of the hole, the position of the distance changes. The bore has a slight deviation. This is sufficient so that the distance of the tactile measurement differs from that of the other sensors. The measurement by means of the tactile sensor would be less significant for the function of this type of component.

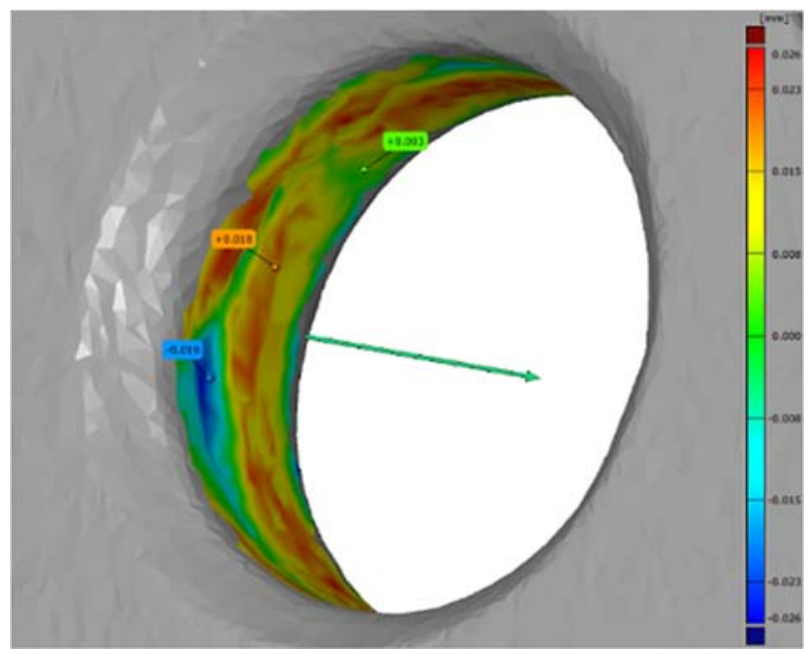

Fig. 1. Shape deviation of the diameter of the black plastic material.

The experiment with the orange plastic part have another result (as shown in Table 4). 
Table 4. Evaluation on the orange plastic material.

\begin{tabular}{|c|c|c|c|}
\hline \multicolumn{4}{|c|}{ Plastic part - Orange / $\mathbf{8 \times}$ Magnification } \\
\hline Illumination / light intensity & $\begin{array}{c}\text { Diameter left } \\
{[\mathrm{mm}]}\end{array}$ & $\begin{array}{c}\text { Diameter right } \\
{[\mathrm{mm}]}\end{array}$ & Distance [mm] \\
\hline Transmitted light = 15\% & 3,356 & 3,364 & 70,257 \\
\hline Transmitted light = 20\% & 3,357 & 3,365 & 70,256 \\
\hline Transmitted light = 27\% & 3,359 & 3,365 & 70,256 \\
\hline Bright field reflected light =5\% & 3,339 & 3,350 & 70,251 \\
\hline Tactile sensor & 3,359 & 3,360 & 70,243 \\
\hline Computed tomography & 3,357 & 3,362 & 70,258 \\
\hline \hline MAX-MiN & 0,020 & 0,015 & 0,015 \\
\hline
\end{tabular}

Differences are caused by the bright field contrast. The different lightning types combined with different materials (bright colors), has the effect that different measurements results are possible. Thus, the influence is caused through the bright field reflection light. The following Figure 2 shown, that the contrast produced in this context rushing effects. The contour image processing calculated associated element by the Gaussian method.

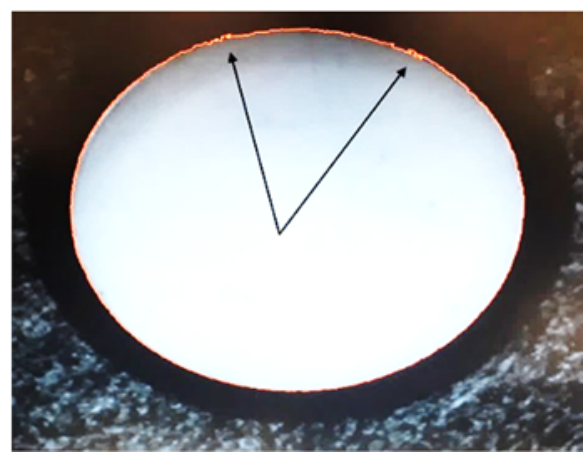

Fig. 2. Systematic measurement influences.

\section{Comparability investigation by combined analyses of surface comparative pattern}

Roughness parameters are calculated from the filtered profile. Therefore, it is important to know which wavelength limit was used, especially for comparative measurements. Basically, the definitions can be applied to the primary profile, waviness and roughness. Depending on the profile, these characteristic values are marked with the capital letters $\mathrm{P}, \mathrm{W}$ or R and the index [5] belonging to the characteristic value. The profile parameters are defined in accordance with [6] the individual measuring section and are therefore first calculated on this section. Unless otherwise stated, the value of a profile parameter is obtained by averaging the individual results of individual measuring sections in direct succession. Modern surface measuring instruments output many different surface parameters which significance is quite different [5]. Profile depth Pt is the distance between two parallel lines that enclose the unfiltered surface profile. The lines have the shape of the ideal profile (e. g. circle, straight line).

This surface characteristic value, as shown in Figure 3, is used for the comparability test of the tactile and laser point sensor, as this is the only usable output of the compared sensors.

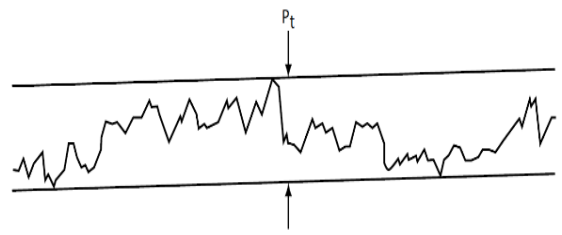

Fig. 3. Derivation of the profile depth $\mathrm{P}_{t}[5]$. 
Tactile and optical measuring devices are used to determine surface parameters. In tactile devices, the surface is traversed with a diamond tip. In contrast, optical measuring devices work contactless. In most cases, optical measuring instruments can resolve a surface much better. In addition, the amplitudes are not influenced by the geometry of the probe tip. Due to these effects, measurement results recorded on different instruments may differ.

The sensors are examined using defined surface comparison patterns classified in VDI 3400. Figure 4 shows surface comparison patterns in different roughness classes and in the colors, black, grey and orange. The images were taken in the live mode of a laser-integrated microscopy.
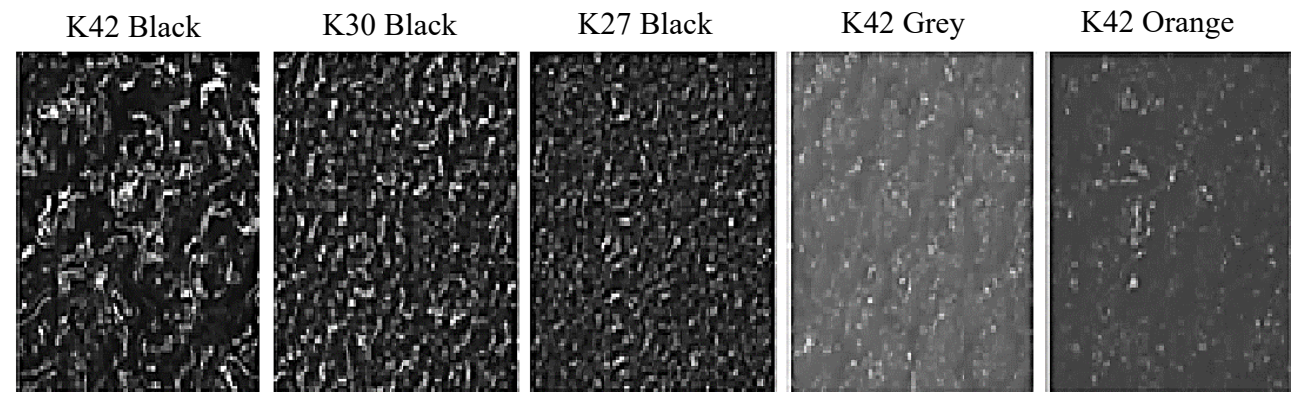

Fig. 4. Surface comparison patterns in different colors and roughness classes.

The following Figure 5 demonstrate a practice example, which is evaluated by a tactile surface scan on the K42 in Black (Figure 4).

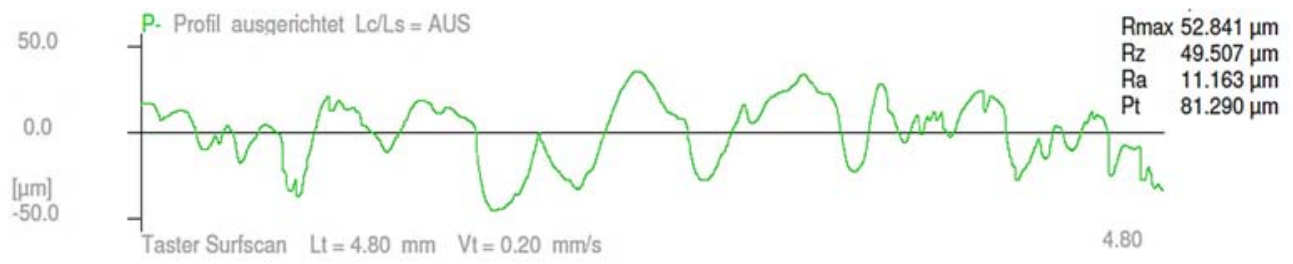

Fig. 5. Tactile surface scan evaluation of the $\mathrm{P}_{\mathrm{t}}$, for the distance of $4.80 \mathrm{~mm}$.

Evaluation of the laser point sensor:

The fixed speed $=0.2 \mathrm{~mm} / \mathrm{sec}$, just like the tactile stylus method. Additionally, a section of $4.80 \mathrm{~mm}$. The following Table 5 shows the evaluation by means of laser point sensor. All values listed in the tables below are mean values from several measurements each, so that also runaways are largely excluded.

Table 5. Evaluation of the laser point sensor.

\begin{tabular}{|c|c|c|c|c|c|}
\hline \multicolumn{6}{|c|}{ Laserpointsensor } \\
\hline \multicolumn{2}{|c|}{ Surfacecolour (Roughness-class / Measurand) } \\
\hline Black (Class / Measurand) & Grey (Class / Measurand) & Orange (Class / Measurand) \\
\hline $\mathrm{K} 42$ & $83 \mu \mathrm{m}$ & $\mathrm{K} 42$ & $64 \mu \mathrm{m}$ & $\mathrm{K} 42$ & $59 \mu \mathrm{m}$ \\
\hline $\mathrm{K} 30$ & $19 \mu \mathrm{m}$ & $\mathrm{K} 30$ & $21 \mu \mathrm{m}$ & $\mathrm{K} 30$ & $18 \mu \mathrm{m}$ \\
\hline $\mathrm{K} 27$ & $15 \mu \mathrm{m}$ & $\mathrm{K} 27$ & $9 \mu \mathrm{m}$ & $\mathrm{K} 27$ & $13 \mu \mathrm{m}$ \\
\hline
\end{tabular}

The direct comparison by means of the tactile profile method, which is also common in the classical industry, is illustrated in Table 6. 
Table 6. Evaluation by tactile profile method for direct comparison with the laser point sensor.

\begin{tabular}{|c|c|c|c|c|c|}
\hline \multicolumn{6}{|c|}{$\begin{array}{l}\text { Reference Principle = Tactile Measurement Principle } \\
\text { Surfacecolour (Roughness-class / Measurand) }\end{array}$} \\
\hline \multicolumn{2}{|c|}{ Black (Class / Measurand) } & \multicolumn{2}{|c|}{ Grey (Class / Measurand) } & \multicolumn{2}{|c|}{ Orange (Class / Measurand) } \\
\hline K42 & $81,29 \mu \mathrm{m}$ & K42 & $95,01 \mu \mathrm{m}$ & K42 & $79,54 \mu \mathrm{m}$ \\
\hline K30 & $20,11 \mu \mathrm{m}$ & K30 & $22,37 \mu \mathrm{m}$ & K30 & $20,62 \mu \mathrm{m}$ \\
\hline K27 & $13,93 \mu \mathrm{m}$ & K27 & $12,10 \mu \mathrm{m}$ & K27 & $14,73 \mu \mathrm{m}$ \\
\hline
\end{tabular}

It can now be said that a good comparability of the sensors can be achieved regarding the black plastic material. The colors grey and orange are different. The contrast decreases sharply here and becomes very blown, so that these materials produce colors and random measurement deviations. Therefore, it can be concluded that the structure of the surface (mountains and valleys) cannot be ideally captured due to the contrast. The metal objects were not analyzed for this investigation, because the laser point sensor create strong reflections and that's why no comparability.

\section{Conclusions}

The comparison of different sensors on different workpiece properties proves that reproducible and comparable measurement results can be achieved depending on the type of application. By taking the experimental findings obtained here into account, not only the users of the measurement technology benefit, also other areas such as the quality engineers, who can avoid typical influences even in the early stages of maturing. In this way, errors can be detected at an early stage and countermeasures can be implemented. Of course, it is first of all important in which order of magnitude the tests are carried out and measured values are given, but here it was important to set determine the best condition. In essence, the experimental investigation has contributed to the optimization and sensitization of the different measuring sensors and to the fact that communication with internal and external partners is facilitated and can now be improved. The aim in the coming years will be to largely increase the degree of automation and to offer the best possible quality and reliable measurement results. The research is a further step in direction of industry 4.0.

\section{References}

1. Production (News portal of the German industry), Quality assurance in the manufacturing (in German) (2015), https://www.produktion.de/technik/automatisierung/qualitaetssicherung-in-der-fertigung-304.html, accessed 12.12.2017

2. DIN EN ISO 8015 (Standard), Concepts, principles and rules (2011)

3. DIN 16742 (Standard), Tolerances and acceptance conditions (2013)

4. R. Christoph, H.-J. Neumann, Computed tomography in the production metrology, South German publisher $2^{\text {th }}$ Edition (in German) (2012)

5. B. Bertsche, W. Haas, S. Jung, Surface assessment, University Stuttgart (in German) (2012)

6. DIN EN ISO 4287 (Standard), Profile method, Terms, definitions and surface texture parameters $(2010)$ 